EDITORIAL

Rev Chil Salud Pública 2014; Vol 18 (3): 245-246

\section{LA SALUD DE LA HISTORIA: DE MORIBUNDOS Y VIVIENTES}

Richard Horton propuso como comentario en julio en The Lancet, ${ }^{1}$ que la producción histórica actual en salud pública está alejada de los problemas presentes. Recordando a algunos notables autores, terminaba citando la vida y obra de Roy Porter, como ejemplo de la fecundidad que una vinculación de campos puede implicar. La coda de su Offline es algo melancólica:

So where are the historians of today to illuminate the past as we struggle with the aggressive commercialisation of medicine, failures of professional leadership, notions of free will and death, misuse of medicines, paralysis in public health policy, or catastrophic failures of care? They appear to have evaporated, leaving a residue of dead and inert dust.

Tal comentario no tardó en ser respondido por Carsten Timmermann ${ }^{5}$ a nombre de los historiadores, quien desplegó algo de la enorme producción historiográfica existente en el área, los sitios e iniciativas en funcionamiento y ofreciendo una columna regular a Lancet, junto con una actualización de la lista de lecturas de Horton.

Paradójicamente en el mismo número se publicó un artículo de Stephen Mawdsley, que a través de una elucidación histórica de los casos de polio provocados por inyecciones, proponía argumentos rotados en 90 grados respecto de lo que ambos interlocutores sugerían. La solución de un problema técnico en las campañas de vacunación de polio encontró en el análisis histórico argumentos de igual peso que las pruebas de laboratorio. Vivian Wyatt, microbiólogo, intrigado por los casos de los años ochenta, buscó en las revistas médicas del siglo XX reportes semejantes a los que en ese momento estaban en debate y pudo sugerir que el problema ya había sido reconocido y examinado en dos oportunidades. La conclusión del articulista no deja de conmovernos, como defensores de archivos, registros y de su apertura al público. ${ }^{2}$

Far from being bibliographic curios, archived medical journals contained the remnants of a neglected theory and important observations relevant to a contemporary concern. Historical record and interpretation ultimately grounded conceptions of polio provocation and added weight to fresh discoveries. By building on knowledge, physicians and public health researchers
Escuela de Salud Pública, Facultad de Medicina Universidad de Chile. ycarvajal@med.uchile.cl 
forged a momentum to solve a mystery, which ultimately belped to reduce suffering and save lives. ${ }^{3}$

Dos mundos que desean e intentan conectarse, que cuestionan el orden actual y la emergencia de barreras conceptuales entre ciencias sociales y medicina. Dos mundos reconociendo la inutilidad de su separación, esbozando un intercambio que conecte y actualice la intimidad entre medicina e historia, pero a la vez volviendo a tejer la separación.

Como editor inquieto por la falsa distinción instalada en el corazón de la salud pública, que separa la epidemiología de las políticas públicas, las ciencias sociales de la medicina, lo social de lo natural, lo poblacional de lo individual, no puedo dejar de pensar en el equívoco de dos mundos, que pese a hablar del mismo problema, lo hacen sin entenderse. Este extraño diálogo, me parece, refleja una obstinada persistencia de los desencuentros que aparecen cada vez que buscamos realizar la vinculación entre especialistas que tememos salir de nuestra especialidad hacia las otras. Historiadores que creen que estudian la historia de la medicina solo en tanto historiadores, dejando de lado los elementos técnicos que son la médula del problema. O clínicos que creen a su vez, que la clínica unicamente contiene en su interior clínica y nada de política, ética, filosofía o historia.

Más que nunca, necesitamos historiadores que lean las revistas de medicina no solo como referencias temporales, sino como el corazón de la disciplina médica. Y clínicos que sepan leer la medicina actual, con una sabiduría que combine reflexividad sociológica y astucia historiográfica. Capaces de moverse en el eje vertical del debate.

Se dirá que no es posible. Pero si no somos capaces de mostrar que entre ambos extremos del cabo hay una proliferación de vínculos en sentido vertical, no es posible reanudar el lazo cortado.

Para los escépticos, sugiero leer el esfuerzo de Stephen Mawdsley por migrar a The Lancet desde su artículo original, ${ }^{3}$ y el del mismo Vivian Wyatt, cuya biología e historiografía resultan indistintamente amalgamadas, ${ }^{6}$ en producciones cargadas de inquietudes historicistas y epistemológicas a la vez. ${ }^{7}$

\section{REFERENCIAS}

1. Richard Horton. Offline: The moribund body of medical history. The Lancet, 384: 292, July 2014.

2. Yuri Carvajal and Silvina Veto. Investigación y patrimonio: carta abierta a CONICYT y DIBAM. Revista Chilena de Salud Pública, 17(3): 234-236, 2013.

3. Stephen Mawdsley. The art of medicine. polio provocation: solving a mystery with the help of history. The Lancet, 384: 300-301, Jyly 26, 2014.

4. Stephen Mawdsley. Balancing Risks: Childhood Inoculations and America's Response to the Provocation of Paralytic Polio. Social History of Medicine, 26(4): 759778, November 2013.

5. Cartsen Timmermann. Not moribund at all! an historian of medicine's response to Richard Horton. http://somatosphere.net/2014/08/not-moribund-at-all-an-historian-of-medicines-response-to-richard-horton.html, August 2014.

6. Vivian Wyatt. Provocation poliomyelitis: neglected clinical observations from 1914 to 1950. Bull Hist Med, 55: 543-557, 1980.

7. Vivian Wyatt. How history has blended. Nature, 249(5460): 803-805, June 28, 1974. 